\title{
LA EXTENSIÓN DEL SEGURO DE SALUD EN IBEROAMÉRICA: UNA ESTRATEGIA DE POLÍTICA EXTERIOR DEL FRANQUISMO EN LA INMEDIATA POSGUERRA (1942-1959)
}

\author{
Margarita Vilar Rodríguez \\ Universidad de A Coruña \\ mvilar@udc.es \\ Jerònia Pons Pons \\ Universidad de Sevilla \\ jpons@us.es
}

Recibido: 28 marzo 2014; Aceptado: 19 septiembre 2014.

Cómo citar este artículo/Citation: Vilar Rodríguez, Margarita y Jerònia Pons Pons (2015), "La extensión del seguro de salud en Iberoamérica: una estrategia de política exterior del franquismo en la inmediata posguerra (1942-1959)", Asclepio 67 (1): p087. doi: http://dx.doi.org/10.3989/asclepio.2015.13

RESUMEN: La dictadura franquista por motivos de estrategia política intentó influir en materia de seguros sociales, en especial en la cobertura de la enfermedad, en los países iberoamericanos durante las décadas de 1940 y 1950. Los resultados de esta colaboración resultaron mediocres en la práctica y produjeron pocos avances reales, al margen de la imitación de las instituciones de gestión y algunos cursos de formación actuarial y estadística. Este fracaso se debió en gran medida al propio atraso y debilidad del modelo de cobertura sanitaria de la dictadura franquista ahogado por un déficit financiero crónico y con graves limitaciones tanto en la cobertura de la población como en las prestaciones ofrecidas. A partir de la década de 1960, una vez superada la etapa de aislamiento y autarquía, las autoridades franquistas redujeron su interés en la estrategia iberoamericana y comenzaron a mirar a Europa.

PALABRAS CLAVE: Seguro de enfermedad; Seguridad social; Iberoamérica; España; Dictadura franquista.

\section{THE EXPANSION OF HEALTH INSURANCE IN LATIN AMERICA: A FOREIGN POLICY STRATEGY OF THE FRANCO REGIME IN THE POST-CIVIL WAR PERIOD (1942-1959)}

ABSTRACT: For reasons of political strategy, the Franco dictatorship sought to have an influence in matters of social insurance, especially sickness coverage, in Latin American countries during the 1940s and 1950s. The results of this collaboration were mediocre in practice and led to little real progress, apart from imitating management institutions and some actuarial and statistical training courses. This failure was due to a large extent to the backwardness and weakness of the Franco dictatorship's own model of health care provision, stifled by a chronic financial deficit and with serious shortcomings in both coverage of the population and provisions offered. Beginning in the 1960s, once the stage of isolation and autarky was overcome, the Francoist authorities reduced their interest in the Latin American strategy and started to look towards Europe.

KEY WORDS: Sickness insurance; Social security; Spain; Latin America; España; Franco dictatorship.

Copyright: () 2015 CSIC. Este es un artículo de acceso abierto distribuido bajo los términos de la licencia Creative Commons Attribution-Non Commercial (by-nc) Spain 3.0. 


\section{INTRODUCCIÓN}

A principios de los años cuarenta España permanecía a gran distancia de las naciones más industrializadas en la adopción de programas de seguros sociales estatales. En realidad, se encontraba más cerca de la situación de los países iberoamericanos que del grupo de países desarrollados que habían sentado las bases del Estado de bienestar antes de la II Guerra mundial ${ }^{1}$. Desde el punto de vista cronológico, se pueden distinguir dos etapas en el proceso de difusión internacional de los seguros sociales (Ritter, 1991; Usui, 1994). Hasta la I Guerra mundial los seguros se difundieron de manera muy lenta y en la mayoría de los casos bajo regímenes de libertad subsidiada. En esta primera etapa solo Francia, Dinamarca y Reino Unido llegaron a cubrir los cuatro riesgos fundamentales (accidentes de trabajo, pensiones, enfermedad y desempleo), aunque con cobertura limitada. Sin embargo, durante el periodo de entreguerras se produjo un desarrollo acelerado en los seguros sociales, sobre todo en los países de la Europa occidental y otros países de colonización europea. La historiografía ha destacado como principales factores de este despegue: el impacto de la I Guerra mundial sobre las demandas sociales, el fortalecimiento del movimiento obrero y el desarrollo de estructuras democráticas en muchos países. Más tarde, la Gran depresión implicó una ampliación de las responsabilidades del Estado. La Social Security Act. Norteamericana, promulgada por Franklin D. Roosevelt en el contexto del New Deal en 1935, supuso quizás la consagración internacional de esta tendencia.

Por otro lado, hay que destacar también la labor de instituciones internacionales como la Organización Internacional del Trabajo (OIT) y la Asociación Internacional de la Seguridad Social (AISS) en la difusión de los seguros sociales². Más adelante, la Carta Atlántica (1941), los Informes Beveridge (1942 y 1944) y la Declaración Universal de los Derechos del Hombre de 1948 confirmaron esta tendencia, según la cuál «toda persona, como miembro de la sociedad tiene derecho a la seguridad social» (art. 22). Finalmente, en el Informe OIT a las Naciones Unidas (1949) se describe la seguridad social como «un anhelo de los pueblos trabajadores del mundo, de que no se repitan los sufrimientos de la gran depresión anterior a la guerra».

En el núcleo de los países pioneros, todos ellos europeos (Reino Unido, Alemania, Dinamarca e Irlanda), a finales de los años treinta ya se habían implantado los cuatro seguros sociales básicos con elevada cobertura real sobre la población (Köhler y Zacher, 1982; Flora, 1983; Ashford, 1989). En un segundo nivel encontramos otro grupo de países europeos que contaban con los cuatro seguros, pero mantenían unas tasas de cobertura moderadas (Noruega, Suecia, Holanda, Bélgica, Italia, Francia, Austria, Luxemburgo y Checoslovaquia).
El resto de países presentaban en el mejor de los casos sistemas incompletos, ya que habían cubierto algunos de los riesgos sociales básicos. Este era el caso de España, que tuvo que esperar hasta después de la Guerra civil española (1936-1939) para implantar un Seguro Obligatorio de Enfermedad. Por entonces, 25 países europeos, 6 países latinoamericanos, Japón y Nueva Zelanda habían logrado aprobar leyes para cubrir este riesgo (Herranz, 2010 y Murray, 2007). De este modo, mientras en la Europa occidental posterior a la segunda posguerra mundial se desarrollaba un Estado del bienestar sostenido en bases democráticas, en España se ponía en marcha un primer Seguro Obligatorio de Enfermedad en 1942, vinculado a los intereses políticos de una dictadura fascista ${ }^{3}$.

Pero ¿cómo trató la dictadura franquista el tema de la cobertura sanitaria en sus leyes fundamentales? Durante la Guerra civil, los principios básicos del gobierno sublevado quedaron definidos en el Fuero del Trabajo (1938). Por lo que se refiere al periodo objeto de estudio en este artículo, solo encontramos dos referencias (Benjumea, 1990). De un lado, los artículos 28 y 29 del Fuero de los españoles (Ley de 17 de julio de 1945) reconocieron el derecho de los trabajadores a la cobertura de los riesgos sociales y el compromiso del Estado para mantener instituciones de asistencia públicas o impulsar la creación de otras privadas. De otro lado, los Principios del Movimiento Nacional (17 de mayo de 1958) ratificaron de nuevo el derecho de los españoles a los «beneficios de la asistencia social y los servicios sociales», aunque no contemplaron el derecho a la salud de manera específica. Sin embargo, el Seguro Obligatorio de Enfermedad aprobado en 1942 se había convertido en la pieza clave de un discurso propagandístico paternalista de «justicia social» que trataba de amortiguar las tensiones internas del país en un marco de control de la población, represión laboral y pésimas condiciones de vida. Nos referimos a un sistema de cobertura imperfecto que generaba graves problemas de equidad derivados de la desigualdad protectora. Por ejemplo, el sistema dejaba fuera de su ámbito de prestaciones a sectores tan amplios de la población trabajadora como los activos agrarios, que representaban el $50,5 \%$ de la población activa total española en 1940 (Nicolau, 2005). Además, el Estado se desentendió por completo de su financiación y se limitó a desempeñar un papel regulador, exigiendo las cuotas a quienes por ley estaban obligados a cotizar al sistema y regulando los pagos cuando el posible beneficiario cumplía las condiciones exigidas por la ley. Por tanto, el régimen franquista defendía en última instancia el principio de autofinanciación del régimen social ${ }^{4}$.

En general, la mayor parte de los seguros sociales durante la dictadura franquista estuvieron costeados básicamente por empresarios y trabajadores, quie- 
nes destinaban un porcentaje de su raquítico salario a las cotizaciones y recibían unas prestaciones irrisorias, cada vez más depreciadas por la fuerte inflación del periodo. En consecuencia, los trabajadores se convirtieron en las principales víctimas de las imperfecciones del sistema de previsión social franquista. Este modelo protector careció además de dos pilares básicos en los modelos de cobertura social vigentes en los países de la Europa occidental tras la II Guerra mundial: la universalización de las prestaciones y el carácter redistributivo desde el punto de vista social (Comín, 1996). Más allá de los fines estratégicos del seguro en el interior del país, la dictadura lo utilizó también como herramienta clave en su política exterior en la inmediata posguerra, tratando de exportar el modelo a las naciones iberoamericanas políticamente más próximas al régimen. Este artículo propone analizar este proceso poco conocido y estudiado en la historiografía española que ofrece una evidencia clara del papel de "Madre Patria» que la dictadura pretendía representar con los países latinoamericanos y un ejemplo de la maquinaria propagandística del régimen que pretendió dar lecciones de un sistema de previsión con graves problemas financieros y con una cobertura muy limitada.

\section{LA POLÍTICA EXTERIOR DEL FRANQUISMO EN TORNO AL SEGURO DE ENFERMEDAD EN LA INMEDIATA POSGUERRA}

La derrota de las potencias del Eje en la II Guerra mundial obligó al franquismo a configurar una nueva estrategia en su política exterior. Sin embargo, los aliados no olvidaron en la inmediata posguerra las simpatías mostradas por la dictadura española a los regímenes derrotados ${ }^{5}$. La desconfianza mutua entre el gobierno franquista y los países capitalistas occidentales se tradujo en aislamiento diplomático y exclusión de algunos organismos internacionales como la ONU. La tensa situación tampoco facilitó los contactos económicos con el extranjero. Tras una década de aislamiento bajo el periodo autárquico que se prolongó hasta los años cincuenta, la dictadura franquista logró que las democracias occidentales pasasen por alto su alianza con el nazismo y lo integrasen en su sistema estratégico, a la sombra de la guerra fría. Así, por ejemplo, Estados Unidos promovió la inclusión de España en la ONU en 1955. Desde esta década, la política económica de la dictadura emprendió el camino de la liberalización económica, aunque sin renunciar a sus bases ideológicas y al control y represión de la población.

Dentro de este contexto, Franco trató de buscar alianzas políticas con los países de América Latina con el fin de que España pudiera volver a representar cierto papel en el marco internacional. Aún siendo consciente de que ya no era posible volver al imperio territorial, la dictadura soñaba con recuperar el papel tutelar, la influencia política y la posición de liderazgo de España sobre los países latinoamericanos ${ }^{6}$. En realidad, el interés del fascismo por convertir a España en "Madre Patria de la Hispanidad» ya aparece en las bases ideológicas de los grupos fascistas, sobre todo la Falange Española, durante la década de 1930. Sin embargo, tras la Guerra civil, la idea de Hispanidad, directamente relacionada con el ansia de ser imperio, cobrará nueva forma, transformándose en un símbolo de la «nueva» España fascista y de su política exterior. En consecuencia, la política iberoamericana pasó a representar un doble papel en la dictadura: un instrumento propagandístico para legitimarla en el poder, al identificarla con la España imperial defensora de los valores católicos, y una herramienta para ganar aliados, influencia exterior y lograr que el país fuese admitido de nuevo en la comunidad internacional. La previsión social representó un papel clave dentro de esta política.

El Ministro de Asuntos Exteriores español, el falangista Alberto Martín Artajo (1945-1957), fue uno de los principales impulsores de la política exterior iberoamericana. Recién llegado al Ministerio impulso la creación del Instituto de Cultura Hispánica (INC) en 1945, con el fin de proyectar la imagen de España y coordinar las relaciones culturales con el mundo hispánico ${ }^{7}$. Durante los años cincuenta, en la sede madrileña del Instituto se multiplicaron las conferencias, seminarios, exposiciones y congresos en torno al tema central de las relaciones entre España e Iberoamérica. A medida que se comenzaron a recuperar los contactos diplomáticos con el bloque occidental, la estrategia iberoamericana dejó de tener un papel central en la política exterior española, aunque el proyecto nunca se abandonó por temor a un nuevo rechazo ${ }^{8}$.

Al igual que otros muchos ámbitos, la política de seguros sociales fue utilizada por la dictadura como "un eslabón más de la cadena que hoy liga a los pueblos de Iberoamérica $»^{9}$. En realidad, los países de la otra orilla del Atlántico ya venían colaborando entre ellos en este ámbito desde 1936, cuando los Estados miembros de la Organización Internacional del Trabajo suscribieron una resolución que establecía los principios fundamentales del derecho de seguro social para los trabajadores en América durante la conferencia de trabajo de Santiago de Chile $^{10}$. Dos años más tarde, quedó constituido un Comité Interamericano de Iniciativas en Materia de Seguridad Social con el propósito de que los gobiernos e instituciones de seguridad social de los países de América llegaran a constituir, con carácter permanente, un organismo de estudio, colaboración, información y acción teórica. El Comité celebró la primera Conferencia Interamericana de Seguridad Social (CISS) en Chile, los días 10 y 16 de septiembre de 1942. A este foro asistieron buena parte de los países del continente: Argenti- 
na, Bolivia, Brasil, Canadá, Colombia, Costa Rica, Cuba, Chile, Ecuador, El Salvador, Estados Unidos, Guatemala, Haití, México, Nicaragua, Panamá, Paraguay, Perú, República Dominicana, y Uruguay, así como representantes de la Oficina Internacional del Trabajo, de la Oficina Sanitaria Panamericana y del Instituto Internacional Americano de Protección a la Infancia. Entre sus principales objetivos podemos destacar: contribuir al desarrollo de la seguridad social en los países de América, cooperar con las instituciones y administraciones de seguridad social; emitir declaraciones, adoptar resoluciones y formular recomendaciones en materia de seguridad social; e impulsar la cooperación e intercambio de experiencias.

La dictadura franquista reconocía a finales de los años cuarenta que estos organismos venían realizando una importante labor en materia social, pero decidieron impulsar su propia red de cooperación con los países de América Latina bajo dos argumentos principales que teóricamente legitimaban su iniciativa. Primero, la ejemplar previsión social española, que describían como un modelo digno de exportar. En consecuencia, la experiencia española podía ser de gran utilidad para países con raíces culturales comunes. Segundo, señalan que la participación de Estados Unidos como país miembro de la CISS suponía la presencia de "dos posiciones teóricas dispares en el terreno de los principios sociales y morales del seguro, lo que podría perjudicar los intereses del mundo hispánico» ${ }^{11}$. Desde el "viejo solar católico de la Madre Hispana», España propuso a estos pueblos un nuevo foro para cooperar en el logro de mejoras sociales, sin el yugo de la América del Norte ${ }^{12}$

El Ministro de Asuntos Exteriores (Martín Artajo) y sobre todo el Ministro de Trabajo (Girón) y los miembros del Instituto Nacional de Previsión (INP) y del Instituto de Cultura Hispánica se entregaron a esta labor durante los años cuarenta, a través de contactos diplomáticos y múltiples viajes por diversos países latinoamericanos. Fruto de esta estrategia, se consiguió celebrar el I Congreso Iberoamericano de Seguridad Social en la sede del INC en Madrid en $1951^{13}$. Su principal objetivo era la cooperación entre los países iberoamericanos de las dos orillas del atlántico en materia de seguros sociales ${ }^{14}$. En dicha reunión estuvieron representados Argentina, Bolivia, Brasil, Colombia, Costa Rica, Cuba, Chile, Ecuador, España, Filipinas, Haití, Paraguay, Perú, Portugal, República Dominicana, El Salvador, Uruguay y Venezuela. Dos ausencias Ilaman poderosamente la atención, Estados Unidos y México, países que no habían reconocido al régimen franquista y con los que éste no mantenía relaciones diplomáticas, lo que pone en evidencia el carácter político de esta iniciativa. Once de dichos países ostentaron delegaciones gubernamentales, ascendiendo el número de participantes en el Congreso a 166 representantes iberoamericanos y 294 españoles. Como observadores, asistieron también enviados especiales de algunos países europeos (p.e. Italia y Bélgica), miembros de la Asociación Internacional de Seguridad, Social y del Comité Iberoamericano de Seguridad Social y se contó con el respaldo explícito de la Oficina Internacional de Trabajo y de la Conferencia de Estados Americanos. ${ }^{15}$ Esta Conferencia resultó un éxito diplomático y político para una dictadura franquista donde coleaban los últimos signos de la autarquía y del aislamiento exterior. El régimen aprovechó este escaparate para difundir propaganda sobre los grandes avances de España en materia de seguros sociales, sin reparar en que por aquellos años la mayoría de su población seguía bajo la soga del racionamiento, pésimas condiciones de trabajo y duras políticas represivas ${ }^{16}$.

No se perfilaron grandes novedades en las conclusiones de este primer Congreso, pero sí consiguió consolidar la iniciativa con la creación del Consejo Asesor de la Oficina Iberoamericana de Seguridad Social (OISS), encargada de organizar seminarios de estudios sobre terminología, legislación comparada y problemas de aplicación práctica de la seguridad social; convocar becas y cursos de formación destinados a profesionales iberoamericanos que deseasen estudiar en España el tema de los seguros sociales; y editar diversas publicaciones formativas y técnicas ${ }^{17}$. El papel hegemónico que pretendía representar España se vio reflejado en los miembros que integraron inicialmente dicho Consejo, todos del mismo país, aunque los cargos serían rotatorios: Fernando Coca (Director General de Previsión) como presidente; Alfredo Sánchez Bella (Director del Instituto de Cultura Hispánica), vicepresidente; y como vocales Luis Jordana de Pozas (represente de la Comisión Iberoamericana de Seguridad Social del Instituto Nacional de Previsión), Hermenegildo Baylos Gorroza y Antonio Pedroso Latas (Instituto Social de la Marina), Antonio Correa Veglism (Servicio Nacional de Montepíos y Mutualidades Laborales) y Joaquín de la Vega Samper (Servicio de Reaseguro de accidentes de trabajo). La Secretaría General con sede en Madrid quedó en manos de Carlos Martí Bufill, miembro del Instituto de Cultura Hispánica desde su fundación, lo que resultó clave para convertirlo en una de las personas más influyentes en la colaboración entre España y Latinoamérica, al menos en el apartado de los seguros sociales.

Carlos Martí Bufill (Girona 1915 - Madrid 2001), abogado de profesión, fue uno de los mayores expertos en materia de seguros sociales iberoamericanos en la España de la época. Ejerció diversos cargos como Jefe Adjunto del Servicio Exterior y Cultural del INP, secretario general del INP, secretario de la Sección de Estudios Sociales del Seminario de Problemas Hispa- 
noamericanos, vinculado al Instituto de Cultura Hispánica, entre otros. Bufill fue uno de los principales impulsores de la OISS. Actuó también como delegado de España en la Asociación Internacional de Seguridad Social en la década de 1960 y su prestigio internacional le condujo a presidir la comisión permanente de esta organización en $1969^{18}$. Fue un hombre polifacético, que realizó también una intensa labor publicista de los seguros sociales, tema sobre el que escribió varias obras como, por ejemplo, La Seguridad Social en los Estados Unidos de América, España y la Seguridad Social hispanoamericana, Presente y futuro del Seguro Social (1948), en el que dedica un capítulo al análisis del seguro social en Hispanoamérica. Esta obra sería el antecedente de su obra de referencia El Seguro Social en Hispanoamérica publicado en 1949.

Bufill permaneció como secretario general del Consejo asesor de la Oficina Iberoamericana de Seguridad Social durante los siguientes tres congresos iberoamericanos de seguridad social con sedes en Lima (1954), Quito (1958) y Bogotá (1964) ${ }^{19}$. En el primero de los foros participaron 21 países Iberoamericanos y 128 congresistas. Fruto de estos primeros congresos se organizaron comisiones de trabajo, donde participaban grupos de técnicos españoles en seguridad social, que visitaron algunos países latinoamericanos como Bolivia y Ecuador con el fin de colaborar con sus gobiernos de cara a implantar o perfeccionar el seguro de enferme$\mathrm{dad}^{20}$. En paralelo, se habían comenzado a celebrar los primeros cursos de cooperación técnica actuarial patrocinado por la Oficina Iberoamericana de Seguridad Social de España; el curso de racionalización y mecanización de administrativos de la Seguridad Social en Bogotá, patrocinado por el Instituto Colombiano de Seguros Sociales; o el curso de estudios sociales iberoamericanos en la Universidad Internacional Menéndez Pelayo, de Santander; a la vez, se comenzó a publicar la Revista "Acción», órgano oficial de la Organización, y valiosos volúmenes de que contienen todos los trabajos presentados a los congresos y cursos de cooperación ${ }^{21}$.

En el II Congreso Iberoamericano de Seguridad Social celebrado en Lima (Perú) en 1954 quedó aprobada la "Carta Constitucional de la OISS» con la asistencia de 21 países y 128 congresistas. Para esta reunión fueron invitados, además de los gobiernos del mundo iberoamericano, otras entidades oficiales encargadas de la gestión de los Seguros Sociales, las Universidades y Escuelas Sociales o de Servicio Social encargadas de la enseñanza de las cuestiones sociales, así como diversos expertos, especialistas y profesores de los países iberoamericanos y filipinos. Asimismo se habían invitado como observadores a miembros de la Organización de Estados Americanos, la Oficina Internacional del Trabajo, la Asociación Internacional de Seguridad Social, el Comité Interamericano de Seguridad Social y la Organización Mundial de la Salud ${ }^{22}$.
Los principales temas que figuraban en la agenda oficial del Congreso versaron en torno a las fórmulas y los resultados logrados en la aplicación de los Seguros Sociales en el medio campesino; los sistemas de financiación de la seguridad social y sus resultados; los problemas y soluciones para la conservación de derechos de los trabajadores emigrantes, los planes de enseñanza general de la seguridad social; y los sistemas para la formación profesional de técnicos ${ }^{23}$. Además, hemos de destacar que fue en este Congreso donde se fundó la Organización Iberoamericana de Seguridad Social, cuya Secretaría General quedó establecida en Madrid y en manos de Carlos Martí Bufill ${ }^{24}$.

Cuando se celebró el Congreso de Quito en 1958, la OISS ya estaba consolidada. En el discurso inaugural ofrecido por el Ministro de Trabajo español Fermín Sanz Orrió (1957-1962) señalaba el éxito de la Organización en la lucha por la justicia social en el mundo iberoamericano y en la colaboración entre países, tanto en el ámbito político como técnico y financiero en materia social ${ }^{25}$. Dentro de la agenda del III Congreso de la OISS destacó el interés por impulsar «la firma de tratados multilaterales de extensión recíproca de los beneficios del seguro social en favor del afiliado que resolviere trasladarse de un país iberoamericano a otro ${ }^{26}$. Entre los acuerdos tomados en Quito, destacaron dos. Primero, el establecimiento de asistencia técnica y de servicios entre todas las instituciones de seguridad social de Iberoamérica y el reconocimiento recíproco de los derechos sociales de los trabajadores de los países que suscribieran el tratado de cooperación ${ }^{27}$. Segundo, la creación del Centro internacional de formación de técnicos de la Organización Iberoamericana de Seguridad, con sede en el Instituto de Cultura Hispánica (Madrid) ${ }^{28}$. En dicho centro, dirigido por Carlos Bufill, se celebraron cursos anuales durante los años sesenta, muy demandados por posgraduados y profesionales de las instituciones de seguridad social de los países Iberoamericanos estudiantes latinoamericanos. El retorno progresivo de España a los organismos internacionales y la recuperación de sus relaciones diplomáticas, permitió a la directiva de la OISS, encabezada por Carlos Bufill, realizar una intensa labor propagandística e institucional de este organismo así como del papel de liderazgo ejercido por España. Como parte de esta estrategia, se estrecharon contactos y se tomaron acuerdos con importantes organismos internacionales como la Organización de Estados Americanos, cuyo jefe de la División del Trabajo (Beryl Frank) visitó Madrid en 1957, o la División Social de la Organización Internacional del Trabajo, que envió representantes a Madrid en 1961 para dar una conferencia a Madrid en 1961 y conocer sobre el terreno la labor desarrollada por el Centro Internacional de Formación de Técnicos de la Seguridad Social ${ }^{29}$. 
En 1961 la directiva de la OISS celebró una reunión extraordinaria en Madrid, impulsada por la «necesidad de una nueva política de seguridad social que responda a la preocupación por la nueva política económica ${ }^{30}$. Por lo que se refiere a España, los cambios de gobierno de 1957 habían eliminado los residuos autárquicos y promovido el proceso hacia la liberalización económica y el saneamiento de los graves desequilibrios internos ${ }^{31}$. El giro económico del país quedó consolidado con el Plan de Estabilización (1959), que supuso la aceptación por parte de la dictadura de la economía de mercado preponderante en el mundo occidental. Por otro lado, los países iberoamericanos se enfrentaban con una coyuntura excepcional de desarrollo económico que debía ser aprovechada para desarrollar los seguros sociales. Dentro de este nuevo marco, surgió una nueva estrategia sostenida en dos ideas básicas. De un lado, se acordó que el derecho de Seguridad Social debía abarcar a la totalidad de la población laboral, considerándose independiente el hecho de encuadramiento en los sistemas de seguridad social. En este caso, se hizo mención especial a los trabajadores del campo, que permanecían desprotegidos en la mayoría de países que había implantado seguros sociales. De otro, se establecieron nuevos criterios para fijar la cuantía de las prestaciones económicas y sanitarias, con el objetivo de cubrir mejor las necesidades de la población ${ }^{32}$.

En el IV Congreso de la OISS celebrado en Bogotá en 1964 se ratificaron estas tendencias. Allí participaron delegaciones de 17 países iberoamericanos, incluyendo España y Filipinas ${ }^{33}$. En la clausura, el por entonces presidente de la República de Colombia, Guillermo León Valencia, reconocía la labor de España en el desarrollo de los seguros sociales en Iberoamerica. En realidad, aunque la cooperación entre las dos orillas del Atlántico en el ámbito de los seguros sociales continuó hasta el final de la dictadura (1939-1975), desde finales de los años sesenta los países latinoamericanos fueron adquiriendo mayor protagonismo en el seno de la OISS ${ }^{34}$.

\section{INFLUENCIA DE ESPAÑA EN EL ESTABLECIMIENTO DE LA COBERTURA DEL RIESGO DE ENFERMEDAD EN IBEROAMÉRICA}

De lo visto hasta el momento, cabe poner sobre la mesa dos cuestiones principales. En primer lugar, ¿estaban los países de América Latina tan atrasados en materia de seguros sociales como para que la dictadura franquista pudiese dar lecciones en esa materia? En segundo lugar, dentro de este marco geográfico, ¿cuáles fueron los países que colaboraron más estrechamente con España en este terreno y por qué? Respecto a la primera cuestión, entre las primeras muestras de seguridad social en
América Latina deben mencionarse las leyes de accidentes de trabajo, donde encontramos como países pioneros a Guatemala (1906); El Salvador y Perú (1911); Argentina, Colombia y Venezuela (1915); Chile, Cuba y Panamá (1916); México (1917); Brasil (1919); o Uruguay (1920). Sin embargo, esta legislación primaria, de reparación de daños frente al riesgo profesional correspondía en la mayoría de los casos a un seguro facultativo y privado, que dejaba al patrono en libertad de asegurarse o no contra el riesgo previsto tanto en compañía comercial, en mutua patronal como en una institución de seguro social ${ }^{35}$. Más adelante, algunos países cambiaron su trayectoria hacia un seguro social integral que incluía diversas contingencias. Si observamos los años de promulgación de las primeras leyes de seguros sociales (accidentes de trabajo, retiro, enfermedad-maternidad y desempleo), constatamos como 18 países habían aprobado las primeras leyes sobre la cobertura del riesgo de accidentes de trabajo antes de que la Guerra civil española terminase; 8 países habían legislado el seguro de pensiones y 8 países disfrutaban de algún tipo de cobertura sobre el riesgo de maternidad y/o enfermedad (Tabla 1). Más tardío fue el establecimiento de normas legales en torno al seguro de desempleo, a excepción de algunos países como Chile, Uruguay - Venezuela. En cualquier caso, América Latina ya contaba con experiencia en el campo de los seguros sociales cuando la dictadura intentó exportar su modelo de previsión social. No obstante, parece que en la mayoría de los países de ultramar los seguros solo cubrían a colectivos profesionales concretos (trabajadores de ferrocarriles, funcionarios públicos...). Esta tendencia comenzó a romperse en los años treinta cuando Ecuador, Chile, Perú y Argentina empezaron a adoptar regímenes generales del seguro social, al menos para algunos riesgos ${ }^{36}$. Poco más tarde, en 1940, Venezuela implantó la Ley integral del seguro social obligatorio contra los riesgos de enfermedad, maternidad, accidentes de trabajo y enfermedades profesionales.

Los progresos en materia social se aceleraron en la década de 1940. La I Conferencia Interamericana de Seguridad Social, celebrada en Chile en 1942 resultó fundamental en este avance ${ }^{37}$. Las resoluciones conocidas con el nombre de "Declaración de Santiago de Chile» apostaban por la ampliación del Seguro Obligatorio de Enfermedad, "de manera que cubra a todos los obreros, empleados, artesanos, profesionales y pequeños comerciantes, así como sus familias. Fruto de estas directrices, la mayor parte de países reformaron sus legislaciones sociales entre 1943 y 1950, ampliando la cobertura y prestaciones o estableciendo un seguro social único e integral. Por el momento, desconocemos el grado de aplicación de esta legislación. 
Tabla 1. Primeras leyes de seguro aprobadas en América Latina

\begin{tabular}{|c|c|c|c|c|c|}
\hline País & Enfermedad & Maternidad & $\begin{array}{c}\text { Accidentes de } \\
\text { trabajo }\end{array}$ & Pensiones & Desempleo \\
\hline Argentina & 1944 & 1934 & 1915 & 1915 & 1967 \\
\hline Bolivia & \multicolumn{2}{|c|}{1949} & 1924 & 1949 & 1953 \\
\hline Brasil & \multicolumn{2}{|c|}{$1934-1936(b)$} & 1919 & $1923(\mathrm{a})$ & 1965 (f) \\
\hline Chile & \multicolumn{2}{|c|}{1924} & 1916 & 1924 & 1937 \\
\hline Colombia & \multicolumn{2}{|c|}{1938} & 1915 & 1946 & 1990 \\
\hline Costa Rica & \multicolumn{2}{|c|}{ 1941-1943 } & 1925 & 1941 & Ninguno \\
\hline Cuba & 1979 & 1934 & 1916 & 1927 & Ninguno \\
\hline Ecuador & \multicolumn{2}{|c|}{1935} & 1921 & 1928 & 1951 \\
\hline El Salvador & \multicolumn{2}{|c|}{ 1949(\#) } & 1949 & $1953-1969$ & Ninguno \\
\hline España & 1944 & (1929) (d) 1931 & 1900 & (1908) (c) 1919 & (1931) \\
\hline Guatemala & 1964 & 1952 & $1906 *$ & 1969 & Ninguno \\
\hline Honduras & 1959 & & 1959 & 1959 & \\
\hline México & 1943 & & 1931 & 1943 & \\
\hline Nicaragua & 1956 & & 1930 & 1956 & \\
\hline Panamá & 1941 & & 1916 & 1941 & \\
\hline Paraguay & 1944 & & 1927 & 1943 & \\
\hline Perú & 1936 & & 1911 & 1936 & \\
\hline $\begin{array}{l}\text { República } \\
\text { Dominicana }\end{array}$ & \multicolumn{2}{|c|}{1947} & 1932 & 1947 & \\
\hline Uruguay & 1960 & 1958 & $1920 * *$ & $1919(\mathrm{e})$ & 1934 \\
\hline Venezuela & 1940 & & $1928(* * *)$ & 1940 & 1940 \\
\hline
\end{tabular}

Fuentes: Martí Bufill (1948); Herranz (2010), p. 63 y Social Security Administration (2009), Social Security Programs Throughout the World: The Americas, SSA.

(a) 1923 (ferrocarriles), 1934 (comercio), y 1936 (industria).

(b) 1923 (ferrocarriles), 1934 (comercio), y 1936 (industria).

(c) Entre paréntesis libertad subsidiada.

(d) 1929 subsidio de maternidad y 1931 seguro obligatorio de maternidad

(e) Aunque entre 1829 y 1934 ya se habían promulgado varias leyes para ciertos grupos de trabajadores.

(f) Se refiere a un fondo de pagos, el seguro de desempleo no se estableció hasta 1986.

$(*)$ en Social Security Administration (2009) pone 1947.

$(* *)$ en Social Security Administration (2009) pone 1914.

$\left({ }^{* * *}\right)$ en Social Security Administration (2009) pone 1923.

(\#) La ley de 29 de septiembre de 1942 fue modificada por la ley de Seguro Social de 28 de septiembre de 1949 y, más tarde, por la nueva ley (decreto de 1263) de 1953. Revista Iberoamericana de Seguridad Social, 1954 (2), p. 301.

A finales de los años cuarenta, 16 países de la comunidad iberoamericana disfrutaban de un sistema de seguro social con tres criterios de aplicación a efectos de personas protegidas: países que centraron su sistema de protección en la población trabajadora considerada económicamente débil (Perú); países que incluyeron en su sistema de seguro social a los trabajadores independiente- mente de la cuantía de sus ingresos (Brasil, Costa Rica, Chile, Ecuador, Argentina, Méjico, Paraguay, Bolivia y el Salvador); o países de sistemas mixtos que combinaron los dos criterios anteriores (Venezuela, Colombia, Panamá y República Dominicana). En este último grupo quedaría enmarcada también España (Pons y Vilar (2011a) y (2011b) y Vilar y Pons (2012)). El predominio del criterio labo- 
ral explicaría en buena medida el hecho de que en la mayoría países latinoamericanos (y también en España) los programas de seguros sociales quedasen inicialmente en manos de sus respectivos $\mathrm{Mi}$ nisterios de Trabajo. Como excepciones a esta regla tenemos a Perú y Brasil ${ }^{38}$. Por lo que se refiere a la financiación, la mayor parte de los seguros sociales en latinoamérica estaban estructurados sobre la base de la contribución tripartita (tabla 2 ) $^{39}$. A diferencia de la España franquista, eran muy escasos los países donde los Estados no participan en las cargas de los Seguros Sociales, bien de forma directa (cuota) o indirecta (subvenciones) ${ }^{40}$. En algunos casos como el boliviano, el Estado creó o reservó impuestos para costear los seguros ${ }^{41}$. En cuanto a la gestión de los seguros sociales, es posible diferenciar dos grupos de países. En el grupo más numeroso encontramos los casos donde predominaba la administración unitaria del seguro social (que coexistía con algunas instituciones particulares) bajo la supervisión ministerial: Bolivia, Colombia, Costa Rica, Chile, Ecuador, El Salvador, Guatemala, Haití, Honduras, México, Nicaragua, Paraguay, Panamá, Perú, República Dominicana y Venezuela. En otros países como Argentina, Brasil, Cuba o Uruguay se mantenía una gestión heterogénea de los seguros bajo diferentes instituciones nacionales, cajas de previsión o compañías comerciales.

Tabla 2. Financiamiento del seguro de enfermedad y sus variantes c. 1955 (en porcentaje)

\begin{tabular}{|c|c|c|c|c|}
\hline \multicolumn{5}{|c|}{ 1. Seguro de enfermedad-maternidad } \\
\hline & Patrono & Trabajador & Estado & Total \\
\hline Bolivia & 5,5 & 2,5 & Impuesto & 8,0 más impuesto \\
\hline Colombia & 4,0 & 2,0 & 2,0 & 8,0 \\
\hline Costa Rica & 3,0 & 3,0 & 1,0 & 7,0 \\
\hline Guatemala & 2,0 & 1,0 & 1,0 & 4,0 \\
\hline Haití & 2,0 & 2,0 & Subvención & 4,0 más subvención \\
\hline México & 4,0 & 2,0 & 2,0 & 8,0 \\
\hline Venezuela & 2,9 & 2,9 & (1) & 5,8 \\
\hline \multicolumn{5}{|c|}{ 2. Seguro de invalidez-vejez-muerte } \\
\hline Costa Rica & 2,5 & 2,5 & 2,5 & 7,5 \\
\hline México & 3,0 & 1,5 & 1,5 & 6,0 \\
\hline \multicolumn{5}{|c|}{ 3. Seguro de enfermedad-maternidad-invalidez-vejez-muerte } \\
\hline Chile & 10,0 & 5,0 & $5,5(2)$ & 20,5 \\
\hline Ecuador & 7,0 & 5,0 & 2,0 & 14,0 \\
\hline Nicaragua & 9,0 & 4,5 & 4,5 & 18,0 \\
\hline Panamá & 4,0 & 4,0 & 0,8 & 8,8 \\
\hline Paraguay & 10,0 & 5,0 & 1,5 & $16,5(3)$ \\
\hline Perú & 6,0 & 3,0 & 2,0 & 11,0 \\
\hline Rep. Dominicana & 5,0 & 2,5 & 1,5 & 9,0 \\
\hline
\end{tabular}

Fuente: Secretaría General del Comité Permanente Interamericano de Seguridad Social (1956: 25)

(1) En Venezuela el Estado paga los gastos de primera instalación y los de administración. El caso de Guatemala dispone una prestación limitada a ala maternidad y a la posible enfermedad derivada del embarazo. En Costa Rica, en zonas donde se da la prestación familiar, los patronos pagan el 5\%; los trabajadores (4\%) y el Estado (2\%). En Ecuador la cuota total es del 4,4\%

(2) Existen además fuertes subsidios fiscales. La elevada cuota de Chile se explica porque está comprendido en ella el coste del Servicio Nacional de Salud.

(3) Está comprendido el financiamiento del riesgo de accidentes de trabajo y de enfermedades profesionales. 
En el ámbito de las prestaciones predominaba la heterogeneidad. En particular, la prestación económica por la incapacidad temporal producida por la enfermedad estaba prevista en la mayoría de países, aunque con desigual grado de protección, entre el 25 y el $100 \%$ del salario, y siempre por periodo de tiempo limitado (tabla 3). En el caso de la maternidad, se contemplaba la ayuda económica y tres tipos de ayuda material: el subsidio por descanso, el subsidio por lactancia y el ajuar o canastilla. En realidad, por lo que se refiere a la cobertura de la enfermedad, la mayoría de las prestaciones establecidas eran de tipo monetario debido a las dificultades técnicas y financieras para poner en marcha una red de instalaciones y servicios sanitarios. A mediados del siglo XX en América Latina había escasez de médicos. Por ejemplo, en Perú ejercían la profesión alrededor de 3.000 médicos, lo que equivalía a 35 médicos por 100.000 habitantes, frente a los 124 médicos por 100.000 habitantes en Gran Bretaña o 71 en Francia ${ }^{42}$. Una situación semejante presentaban el resto de países latinoamericanos. El número reducido de profesionales se veía agravado por su concentración en las principales ciudades y la falta de interés económico y profesional en ejercer en las zonas apartadas de los respectivos países. En consecuencia, parece claro que existía en todos estos países una gran diferencia entre el avance en el marco legislativo y su aplicación real.

Tabla 3. Las prestaciones de enfermedad y maternidad en Latinoamérica a mediados de los años 1940s

\begin{tabular}{|c|c|c|}
\hline Países & Enfermedad & Maternidad \\
\hline Argentina & $\begin{array}{l}\text { Subsidio medicina preventi- } \\
\text { va, salario íntegro, máximo } 6 \\
\text { meses }\end{array}$ & $\begin{array}{l}\text { Subsidio: } 2 \text { meses y medio de salario: tope } 200 \text { pesos; } 30 \text { días } \\
\text { antes y } 45 \text { días después. } \\
\text { Subsidio complementario: } 100 \text { pesos por hijo y } 20 \text { pesos por cada } \\
\text { subsidio anterior } \\
\text { Canastilla: valor } 40 \text { pesos }\end{array}$ \\
\hline Bolivia & - & - \\
\hline Brasil & $\begin{array}{l}\text { No serán inferiores al } 70 \% \text { del } \\
\text { salario mínimo regional }\end{array}$ & - \\
\hline Colombia & $\begin{array}{l}\text { 66\%; periodo de carencia, } 3 \\
\text { días, sino dura 7; si dura más, } \\
\text { desde el primero; periodo } \\
\text { máximo, } 180 \text { días }\end{array}$ & $\begin{array}{l}100 \% \text { del salario, cuatro semanas antes y cuatro después. Subsi- } \\
\text { dio de lactancia }\end{array}$ \\
\hline Costa Rica & $\begin{array}{l}50 \% \text { del último salario mensual; } \\
\text { periodo de carencia, } 4 \text { días; } \\
\text { duración, } 25 \text { semanas }\end{array}$ & - \\
\hline Cuba & - & $\begin{array}{l}\text { Asegurada: un subsidio variable, de } 1,25 \text { a } 4 \text { pesos, según el suel- } \\
\text { do, durante seis semanas anteriores y seis posteriores al alumbra- } \\
\text { miento. Periodo de espera, cinco meses de cotización durante los } \\
\text { dos últimos años. } \\
\text { Mujer del asegurado: } 25 \text { pesos, si el marido tiene } 10 \text { meses de } \\
\text { afiliación }\end{array}$ \\
\hline Chile & $\begin{array}{l}100 \%, \text { primera semana; } 50 \% \text {, } \\
\text { la segunda; } 25 \% \text {, las restantes. } \\
\text { Periodo de carencia, } 5 \text { días, si } \\
\text { dura menos de } 7\end{array}$ & $\begin{array}{l}50 \% \text {, dos semanas antes y dos después, Lactancia, un cuarto del } \\
\text { salario durante } 8 \text { meses }\end{array}$ \\
\hline Ecuador & $\begin{array}{l}50 \%, \text { cuatro semanas y } 40 \% \text { las } \\
21 \text { restantes }\end{array}$ & $75 \%$, tres semanas antes y cuatro después. Canastilla maternal \\
\hline España & $\begin{array}{l}50 \% \text {, veintiséis semanas, si dura } \\
7 \text { días. Plazo de carencia, } 4 \text { días }\end{array}$ & $\begin{array}{l}60 \% \text {, seis semanas antes y seis después. Lactancia } 7 \text { pesetas por } \\
\text { semana y por hijo }\end{array}$ \\
\hline Filipinas & - & - \\
\hline Guatemala & No determinado & Indemnización y lactancia, no determinado \\
\hline Haití & $\begin{array}{l}\text { Del } 50 \text { al } 75 \% \text { del salario duran- } \\
\text { te veintiséis semanas }\end{array}$ & Subsidio \\
\hline
\end{tabular}




\begin{tabular}{|c|c|c|}
\hline Países & Enfermedad & Maternidad \\
\hline Honduras & - & - \\
\hline Méjico & $\begin{array}{l}\text { Subsidio de 0,64 a 10,56 pesos, } \\
\text { durante veintiséis semanas y a } \\
\text { partir del séptimo día }\end{array}$ & $\begin{array}{l}\text { Un subsidio igual al de enfermedad, durante seis semanas antes } \\
\text { y seis después. Mejorado, } 8 \text { días antes y } 30 \text { después, hasta com- } \\
\text { pletar el salario integro: Lactancia, un subsidio que no exceda del } \\
80 \%\end{array}$ \\
\hline Nicaragua & - & - \\
\hline Panamá & - & $50 \%$, seis semanas antes y después \\
\hline Paraguay & $\begin{array}{l}40 \% \text {, durante seis meses, } \\
\text { prorrogables a doce. Periodo de } \\
\text { carencia, } 6 \text { días }\end{array}$ & $40 \%$, veintiún días antes y cuarenta después. Suministro de leche. \\
\hline Perú & $\begin{array}{l}50 \% \text {, durante cuatro semanas; } \\
40 \% \text {, las restantes. Desde el } \\
\text { tercer días y durante cincuenta } \\
\text { y dos semanas }\end{array}$ & $\begin{array}{l}50 \% \text {, treinta y seis días antes y treinta y seis después. Suministro } \\
\text { de leche durante ocho meses }\end{array}$ \\
\hline $\begin{array}{l}\text { República } \\
\text { Dominicana }\end{array}$ & $\begin{array}{l}50 \% \text { durante veintiséis sema- } \\
\text { nas, después del sexto día }\end{array}$ & $\begin{array}{l}50 \% \text {, seis semanas antes y seis después. Lactancia, durante ocho } \\
\text { meses, el } 10 \% \text {, o suministro e leche }\end{array}$ \\
\hline El Salvador & $\begin{array}{l}75 \% \text { desde el tercer día, hasta } \\
\text { veintiséis semanas }\end{array}$ & $100 \%$, seis semanas antes y seis después. Subsidio de lactancia. \\
\hline Uruguay & - & - \\
\hline Venezuela & $\begin{array}{l}66 \% \text { del salario base diario, des- } \\
\text { de el cuarto día, hasta veintiséis } \\
\text { semanas }\end{array}$ & Igual que enfermedad, $66 \%$, seis semanas antes y seis después \\
\hline
\end{tabular}

Fuente: Elaborado a partir de Martí Bufill (1948), apéndice.

Respecto a la segunda cuestión que nos planteamos sobre qué países colaboraron especialmente con España en el ámbito de los seguros sociales, hemos de destacar siete: Colombia, Bolivia, Ecuador, Honduras, Paraguay, Perú y Nicaragua. A estas alturas de la investigación podemos apuntar dos razones que justificaron la mayor aproximación a este grupo de países. En primer lugar, la dictadura franquista estableció contactos con aquellos Estados que introdujeron la mayor parte de los programas de seguros sociales, especialmente en el caso del riesgo de enfermedad, de manera tardía, solo a partir de los años cuarenta y cincuenta (tabla 1). Se trataba, en general, del grupo de países con la renta más baja en América Latina durante aquellos años, donde la cobertura médica era escasa en la práctica y estaba mal coordinada, cubriendo solo a una pequeña parte de su población.

Dentro del grupo retrasado, Ecuador, Paraguay y Perú fueron más precoces al legislar el riesgo de enfermedad. En 1935 Ecuador puso en marcha el Servicio médico del seguro social, que ofrecía prestaciones enfermedad y maternidad, aunque su cobertura resultó limitada ${ }^{43}$. Más tarde, en julio de 1942 se aprobó la Ley del seguro social obligatorio que cubría el seguro de enfermedad y maternidad, invalidez, vejez, viudedad y orfandad y accidentes de trabajo y enfermedades profesionales. Paraguay creó el Ministerio de Salud Pública y Bienestar Social en 1936 y el Instituto de Previsión Social en 1943, pero hasta 1950 no amplió considerablemente los servicios sanitarios para los asegurados ${ }^{44}$. En 1952, el decreto-ley n. 1860 modificó la ley anterior e introdujo la cobertura de los riesgos de enfermedad no profesional, maternidad, accidentes de trabajo, enfermedades profesionales, invalidez, vejez y muerte de los trabajadores asalariados $^{45}$. Perú legisló pronto el riesgo de enfermedad, pero tardó en extender su aplicación. Primero, creó la Dirección General de salubridad en 1903 y más tarde, en 1935, se fundó el Ministerio de Salud Pública, Trabajo y Previsión Social. Un año después, en 1936, quedó establecido un seguro obligatorio de salud para obreros y trabajadores domésticos. Finalmente, en 1948, se creó el seguro social del empleado y se abrió el Hospital del Empleado. El Sistema Nacional de Servicios de Salud, el Consejo Nacional de Salud y los Consejos Regionales para coordinar su puesta en funcionamiento no quedaron establecidos hasta $1978^{46}$. Entre los países más tardíos en aplicar programas de seguros social encontramos a Nicaragua que aprobó 
su Ley de seguridad social en 1955, aunque hasta 1979 no se creó el Sistema Nacional Único de Salud ${ }^{47}$. Poco tiempo después, Honduras, promulgó la Ley de seguro social (1959), a la vez que estableció el Instituto Hondureño de Seguridad Social, cuya responsabilidad descansaba en garantizar el derecho humano a la salud y a la asistencia médica ${ }^{48}$.

En el caso de Colombia prevaleció el modelo higienista hasta 1950. Bajo este modelo la provisión de salubridad pública se limitaba a atender aspectos de carácter sanitario, mientras que la atención curativa y preventiva en salud tenía que ser financiada por los propios usuarios o por algunas instituciones religiosas de caridad $^{49}$. A mediados de los años cuarenta comenzó a cambiar la política sanitaria con la creación de la Caja Nacional de Previsión que atendía la salud de los empleados públicos y el Instituto de Seguros Sociales, que atendía a los empleados del sector privado forma ${ }^{50}$.

El segundo factor que influyó en la elección del grupo de países resultó determinante: el régimen político vigente y su grado de identificación con la dictadura franquista española ${ }^{51}$. Los casos más claros son los de Colombia, Perú y Nicaragua. Por otra parte, el resto de países vivieron en su mayoría una etapa de inestabilidad política, donde se alternaban breves periodos democráticos con revoluciones y dictaduras militares. En este estadio de la investigación, al margen de la influencia en los organismos iberoamericanos de seguridad social ya explicitada, es difícil determinar la influencia directa del modelo franquista en la puesta en práctica del seguro de enfermedad en estos últimos países. Sin embargo, hay constancia de la colaboración técnica en la puesta en marcha del seguro de enfermedad en el caso boliviano. Aunque Bolivia aprobó una Ley de seguro social general en 1949 y una Ley de riesgos profesionales en 1950, estas leyes no se pusieron en marcha de forma inmediata. La Caja Nacional del seguro social solicitó una Misión Técnica española en 1951. La delegación técnica española fue enviada por la Oficina Iberoamericana de Seguridad Social. Estaba compuesta por un actuario, un médico social, un técnico contable-administrativo y presidida por un jurista ${ }^{52}$. Esta comisión trabajó durante tres meses en la Paz, preparó un texto unificado de las leyes anteriores eliminando aquellos contenidos que impedían su aplicación y prepararon las bases para la puesta en práctica, de forma gradual y progresiva, en especial del seguro de enfermedad y maternidad. Al margen de esta influencia directa, es evidente que muchos de estos países adoptaron elementos de los seguros sociales españoles como la creación de un instituto autónomo que gestionara los seguros de forma similar al Instituto Nacional de Previsión español o la creación de cajas que gestionaban cada seguro. En el caso boliviano este organismo fue la Caja Nacional de seguro social, en el paraguayo el Instituto de Previsión Social o en el caso ecuatoriano el Instituto Nacional de Previsión del Ecuador ${ }^{53}$. Finalmente, la colaboración con los países señalados fue tan estrecha que fructificó en la firma de los primeros convenios generales sobre seguridad social con España en los años 1960. Este fue el caso, por ejemplo, de Ecuador y Paraguay ${ }^{54}$.

\section{CONCLUSIONES}

La España franquista impulso la colaboración iberoamericana en materia de seguros sociales como parte de la estrategia para salir del aislamiento internacional al que se vio sometida en los años posteriores a la II Guerra mundial tras su colaboración con los regímenes fascistas. Con este fin, pretendió extender su modelo de previsión social, construido en base a unos planteamientos poco democráticos, sin un sistema de redistribución de la riqueza y basado en un modelo de cotización de gran coste para la mayoría de trabajadores por los países latinoamericanos. Su influencia se basó en la creación de una nueva institución, la OISS, que mantuvo bajo su control mediante el establecimiento de sus organismos administrativos en Madrid y su dirección en manos de figuras políticas del régimen.

A pesar de que la mayoría de países iberoamericanos fueron miembros de la OISS y que participaron en los diferentes congresos celebrados durante las décadas de 1950 y 1960, la colaboraron bilateral de España en este ámbito resultó más cercana con aquellos Estados con un sistema de seguridad social más atrasado (Bolivia, Ecuador, Honduras, Paraguay, Colombia Perú y Nicaragua) y, en especial, con los tres últimos, cuyo régimen político dictatorial se encontraba más cercano a los planteamientos de la dictadura española.

A finales de los años sesenta, los resultados de esta colaboración se mostraban mediocres en la práctica y habían producido pocos avances en los países iberoamericanos, al margen de la imitación de las instituciones de gestión y algunos cursos de formación actuarial y estadística. Esta escasa influencia real se debió en gran medida a las limitaciones y deficiencias del propio sistema de seguros sociales implantado en España que servía poco de modelo, puesto que estaba inmerso en sus propios problemas. Basta recordar el déficit financiero crónico y la escasez de infraestructuras, derivados del sistema de cotización y de la falta de aportación estatal, o la limitada capacidad de cobertura tanto en porcentaje de población como en prestaciones. A mediados de los años sesenta, una vez integrada en los organismos internacionales de la mano de Estados Unidos, el interés de España por mantener la estrecha colaboración en materia de seguridad social con los países latinoamericanos disminuyó. Las autoridades volvieron la mirada hacia los países europeos e intentaron conseguir acuerdos de protección social para los emigrantes españoles en Europa. 


\section{NOTAS}

1 Mesa-Lago (2010, p. 317) establece tres grupos de países en la puesta en marcha de los primeros programas de seguros sociales en América Latina. El grupo pionero (Uruguay, Argentina, Chile, Cuba, Brasil y Costa Rica - este último dudoso-) comenzó a introducir sus seguros sociales en las décadas de 1920 y 1930; el grupo intermedio (Panamá, México, Perú, Colombia, Bolivia, Ecuador y Venezuela) actuó en materia social en los años cuarenta y cincuenta; y el grupo retrasado (Paraguay, República Dominicana, Guatemala, El Salvador, Nicaragua, Honduras y Haití) que impulsó sus programas de seguros sociales a partir de 1960. Para el seguro de enfermedad, se puede encontrar una clasificación similar en Zschock (1986, pp. 108-109).

2 Rodgers, Swepston, Lee y van Daele (2009). Los seguros sociales se internacionalizaron considerablemente tras el final de la I Guerra mundial, tendencia a la que contribuyó la creación de la OIT. No obstante, antes de 1914 ya existía una activa vida internacional en ese terreno. Cabe mencionar a tres organizaciones: el Comité Permanente Internacional de los Seguros Sociales (CPISS), la Asociación Internacional para la Protección Legal de los Trabajadores (AIPLT), llamada, en general, Asociación de Basilea, y la Asociación Internacional de Lucha contra el Desempleo (AILC) (Comité Permanente Internacional de los Seguros Sociales, 1913), Guinand (2008, pp. 93-113).

3 Sobre estas cuestiones, véanse González Murillo (2008) y Pons y Vilar (2012).

4 Un modelo de corte similar ya se había aplicado en la Italia de Mussolini, de donde el franquismo copió algunos aspectos, véase Molinero (2005, p. 126). Sobre el origen, gestión, prestaciones y financiación del SOE, véanse Pons y Vilar (2011a) y (2011b) y Vilar y Pons (2012).

5 Espadas (1988, p. 173) y Tusell, Avilés, Avilés y Pardo (2000, p. 48 y ss). Véanse también Portero (1989) y Tusell (1989).

6 Arenal (1994, p. 31) y Tusell, Avilés, Avilés y Pardo (2000, p. 309).

7 Boletín Oficial del Estado (BOE) 26/09/1946, p. 7223.

8 Arenal (1994, p. 52).

9 La Vanguardia (Barcelona) (de aquí en adelante (LVG)) 5 de junio de 1951 , p. 14

10 La información sobre la Conferencia Interamericana de Seguridad Social ha sido obtenida de http://www.ciss.org.mx/index_ es.php?mod=historia. Sobre los orígenes de esta organización véase Martí Bufill (1948, pp. 193-198).

11 Martí Bufill (1948, pp. 199-200).

12 LVG (Barcelona) 24 de mayo de 1951, p. 3.

13 El Congreso se clausuró en Barcelona. http://www.oiss.org/ spip.php?article776, LVG (Barcelona) 3 de junio de 1951 y ABC (Madrid), 4 de abril de 1952.

14 http://www.oiss.org/spip.php?article776, LVG (Barcelona) 3 de junio de 1951 y ABC (Madrid), 4 de abril de 1952.
15 LVG (Barcelona) 18 de julio de 1952, p. 5

16 Sobre estos temas, véase Vilar (2009).

17 LVG (Barcelona) 5 de junio de 1951, p. 14 y LVG (Barcelona) 20 de mayo de 1951, p. 4.

18 ABC (Madrid) 14 de noviembre de 1961 y ABC (Madrid) 1 de enero de 1969.

19 ABC (Madrid), 28 de Octubre de 1954, ABC (Madrid) 27 de noviembre de 1958 y ABC (Madrid) 10 de abril de 1964.

20 ABC (Madrid) 17 de julio de 1951, p. 14

21 LVG (Barcelona) 10 de octubre de 1952, p. 4; Revista Iberoamericana de Seguridad Social (1952), II, p. 360; Revista Iberoamericana de Seguridad Social (1952), III, p. 567; Revista Iberoamericana de Seguridad Social (1956), VI, p. 1436 y ss.

22 LVG (Barcelona) 4 de septiembre de 1954, p. 7. Precisamente, España había entrado a formar parte de la Organización Mundial de la Salud en 1951, LVG (Barcelona) 26 de mayo de 1951, p. 3.

23 LVG (Barcelona) 4 de septiembre de 1954, p. 7.

24 LVG (Barcelona) 28 de octubre de 1954, p. 11.

25 LVG (Barcelona) 23 de noviembre de 1958, p. 17.

26 ABC (Madrid) 27 de noviembre de 1958 y LVG (Barcelona) 10 de diciembre de 1958, p. 4.

27 LVG (Barcelona) 10 de diciembre de 1958, p. 4.

28 ABC (Madrid) 5 de noviembre de 1959 y ABC (Barcelona) 7 de febrero de 1959 , p. 9

29 ABC (Madrid) 19 de mayo de 1961.

30 ABC (Madrid) 17 de septiembre de 1961.

31 Sobre la economía del desarrollismo, véase Barciela, López, Melgarejo y Miranda (2001, p. 179 y p. 254).

32 ABC (Madrid) 17 de septiembre de 1961.

33 ABC (Madrid) 10 de abril de 1964. Filipinas es uno de los países con mayor atraso en la introducción de los seguros sociales, y en particular del seguro de enfermedad. La primera Ley de seguridad social es del 20 de mayo de 1954. En ella se introducía la cobertura de las contingencias de vejez, invalidez, muerte, enfermedad y paro pero únicamente para las grandes empresas con más de 200 trabajadores. Revista Iberoamericana de Seguridad Social, 1955 (2), pp. 53-54. Una Ley de 18 de junio de 1954 estudiaba implantar un régimen de seguridad social experimental en algunas zonas del país. Revista Iberoamericana de Seguridad Social, 1955 (3), pp. 437-453.

34 ABC (Madrid) 10 de febrero de 1966. 
35 Secretaría General del Comité Permanente Interamericano de Seguridad Social (1956, p. 11)

36 Sobre estos aspectos, véase Martí Bufill (1948, pp. 32-33) y Revista Iberoamericana de Seguridad Social, 1952 (4), pp. 777-814.

37 Martí Bufill (1948, p. 57 y ss).

38 Zschock (1986, p. 104).

39 Secretaría General del Comité Permanente Interamericano de Seguridad Social (1956, p. 25).

40 Martí Bufill (1948, pp. 115-116)

41 En especial se crearon unos impuestos sobre las exportaciones de mineral (de estaño y de otros minerales) para costear las construcciones e instalaciones necesarias para el funcionamiento de los servicios médicos del seguro general. Revista Iberoamericana de Seguridad Social, 1952 (1), pp. 51-92. En el caso ecuatoriano, el Estado contribuyó a la financiación del seguro de enfermedad, destinando los ingresos de las sucesiones intestadas desde el tercer grado colateral de consanguinidad, el $30 \%$ del producto de los impuestos a las herencias, legado, donaciones, etc y otros impuestos que gravaban las rentas. Revista Iberoamericana de Seguridad Social, 1952 (84), pp. 709-725.

42 Zapata Ballón (1956, p. 22).

43 Giedion, Villar y Avila (2010, pp. 150-151).

44 Este país aprobó en 1980 un código sanitario y en 1996 el Sistema Nacional de Salud, véase Giedion, Villar y Avila (2010, p. 258).

45 Excepto los funcionarios públicos, trabajadores independientes y empleados y obreros del Ferrocarril Central del Paraguay que tenían ya una caja propia. Revista Iberoamericana de Seguridad social, 1952 (3), pp. 589-613.

46 Giedion, Villar y Avila (2010, p. 276)
47 Muiser, Sáenz y Bermúdez (2011).

48 Giedion, Villar y Avila (2010, p. 197) y Bermúdez-Madriz, Sáenz, Muiser y Acosta (2011)

49 Giedion, Villar y Avila (2010, p. 112)

50 Más tarde, en un segundo periodo, entre 1970-1989 se creó el Sistema Nacional de Salud bajo el esquema de subsidios a la oferta en el que los recursos del gobierno central para la salud eran transferidos directamente a la red de instituciones públicas hospitalarias. También se crea un esquema tripartito (Estadoempleadores-empleados) de financiación para la prestación de los servicios de salud a la población trabajadora formal; sin embargo, tal sistema seguía sin proporcionar una atención integral de salud a la población de escasos recursos. Véase, Giedion, Villar y Avila (2010, p. 112).

51 La evolución política de estos países en Bethell (ed.) (2002a) (2000b) y (2002c)

52 La delegación técnica española estaba constituida por Carlos Martí Bufill, secretario de la oficina y Jefe de la Misión, Francisco de Ipiña, Jefe de la Asesoría Actuarial del Instituto Nacional de previsión; Germán Garnacho, Inspector médico nacional del seguro de enfermedad y don Ramón Artigau, administrador de Instituciones sanitarias del seguro de enfermedad. Revista Iberoamericana de Seguridad Social, 1952 (1), pp. 51-92.

53 Sobre la gestión del seguro de enfermedad en el caso boliviano puede consultarse Revista Iberoamericana de Seguridad Social, 1952 (1), pp. 51-92 y en caso de Paraguay en Revista Iberoamericana de Seguridad Social 1952 (39), pp. 589-613. En el caso ecuatoriano, el Instituto Nacional de Previsión debía aplicar el régimen del seguro de enfermedad mediante la Caja del seguro. Esta caja junto con la otra existente, la Caja de Pensiones, era autónoma aunque el Ministerio de Previsión Social y Trabajo ejercía vigilancia sobre ellas. Revista Iberoamericana de Seguridad Social, 1952 (4), pp. 709-725.

54 BOE 18/04/1960, p. 5023 y BOE 23/10/1962, p. 14954.

\section{BIBLIOGRAFÍA}

Arenal, Celestino del (1994), Política exterior de España hacia Iberoamérica, Madrid, Editorial Complutense.

Ashford, Douglas E. (1989), La Aparición de los Estados de Bienestar, Madrid, Ministerio de Trabajo y Seguridad Social.

Barciela, Carlos; López, M. Inmaculada; Melgarejo, Joaquín; y José. A. Miranda (2001), La España de Franco (1939-1975), Madrid, Síntesis.

Benjumea Pino, Pedro (1990), "Sanidad y desempleo". En: VVAA, Historia de la acción social pública en España. Beneficencia y Previsión, Madrid, Ministerio de Trabajo y Seguridad Social, pp. 449-71.
Bermúdez-Madriz, Juan L.; M. Rocío Sáenz; Jorine Muiser; y Mónica Acosta (2011), "Sistema de salud de Honduras", Salud Publica Mex, 53 supl 2, pp. 209-S219.

Bethell, Leslie (ed.) (2002a), Historia de América Latina. 14 América Central desde 1930, Barcelona, Cambridge University Press-Critica.

Bethell, Leslie (ed.) (2002b), Historia de América Latina. 15. El Conosur desde 1930, Barcelona, Cambridge University Press-Critica.

Bethell, Leslie (ed.) (2002c), Historia de América Latina. 16. Los países andinos desde 1930, Barcelona, Cambridge University Press-Critica. 
Comín Comín, Francisco (1996), Historia de la Hacienda Pública II. España (1808-1995), Barcelona, Crítica.

Espadas Burgos, Manuel (1988), Franquismo y política exterior, Madrid, Rialp.

Flora, Peter (1983), State, Economy, and Society in Western Europe 1815-1975: A Data Handbook in two Volumes, Frankfurt, New York: Campus; London: Macmillan Press; Chicago: St. James Press.

Giedion, Ursula; Manuela Villar; y Adriana Avila (2010), Los sistemas de salud en Latinoamérica y el papel del seguro privado, Madrid, Fundación MAPFRE.

González Murillo, Pedro (2008),"El franquismo social; propaganda y seguros a través del Instituto Nacional de Previsión (19391962)". En: Castillo, Santiago (ed.), Solidaridad, seguridad y bienestar. Cien años de protección social en España, Madrid, Ministerio de Trabajo e Inmigración.

Guinand, Cédric (2008), "La creación de la AISS y la OIT", Revista Internacional de Seguridad Social, 61 (1), pp. 93-113.

Herranz, Alfonso (2010), "La difusión internacional de los seguros sociales antes de 1945" En: Pons Pons, Jerònia y Silvestre Rodríguez, Javier (ed.), Los orígenes del Estado del Bienestar en España, 1900-1945: los seguros de accidentes, vejez, desempleo y enfermedad, Zaragoza, Prensas Universitarias de Zaragoza, pp. 51-84.

Köhler, Peter A. y Zacher, Hans F. (ed.) (1982), The Evolution of Social Insurance 1881-1981: Studies of Germany, France, Great Britain, Austria, and Switzerland, New York, St. Martin's Press.

Martí Bufill, Carlos (1948), El seguro social en Hispanoamérica, Madrid, Seminario de Problemas Hispanoamericanos.

Mesa-Lago, Carmelo (2010), "History of Social Security in Latin America". En: VVAA, Encuentro Internacional sobre la Historia del Seguro, Madrid, Fundación MAPFRE-Instituto de Ciencias del Seguro, pp. 309-348.

Molinero, Carme (2005), La captación de las masas. Política social y propaganda en el régimen franquista, Madrid, Cátedra.

Muiser Jorine, M. Rocío Sáenz y Juan L. Bermúdez (2011), "Sistema de salud de Nicaragua”, Salud Publica Mex, 53 supl 2, pp. 233-242.

Murray, John E. (2007), Origins of American Health Insurance. A History of Industrial Sickness Funds, New Haven \& London, Yale University Press.

Nicolau, Roser (2005), "Población salud y actividad". En: Albert Carreras y Xavier Tafunell (coord.), Estadísticas Históricas de España. Siglos XIX y XX, Madrid, Fundación BBVA, pp. 79-154.
Pons Pons, Jerònia y Margarita Vilar Rodríguez (2011a), "Friendly societies, commercial insurance and the state in sickness risk coverage: the case of Spain (1880-1944)", International Review of Social History 56 (1), pp. 71-101.

Pons Pons, Jerònia y Margarita Vilar Rodríguez (2011b), “Labour repression and social justice in Franco's Spain: the political objectives of compulsory sickness insurance (1942-1957)", Labor History, 53 (2), pp. 245-267.

Portero, Florentino (1989), Franco aislado: la cuestión española: 1945-1950, Madrid, Aguilar DL.

Ritter, Gerhard A. (1991), El Estado Social, su origen y desarrollo en una comparación internacional, Madrid, Centro de Publicaciones Ministerio de Trabajo y Seguridad Social.

Rodgers, Gerry, Eddy Lee, Lee Swepston y Jasmien Van Daele (2009), La Organización Internacional del Trabajo y la lucha por la justicia social, 1919-2009, Ginebra, OIT.

Secretaría General del Comité Permanente Interamericano de Seguridad Social (ed.) (1956), Legislación comparada de los seguros sociales en América latina, México, Conferencia Interamericana de Seguridad Social.

Tusell, Javier (1989), La España de Franco: el poder, la oposición y la política exterior durante el franquismo, Madrid, Historia 16.

Tusell, Javier; Juan Avilés Farré; Juan Avilés y Rosa Pardo (2000), La política exterior de España en el siglo XX, Madrid, Editorial Biblioteca Nueva S.L.

Usui, Chikako (1994), "Welfare State Development in a World System Context: Event History Analysis of First Social Insurance Legislation among 60 countries, 1880-1960". En: Thomas Janoski and Alexander M. Hicks (eds.), The Comparative Political Economy of the Welfare State, Cambridge University Press, pp. 254-77.

Vilar Rodríguez, Margarita (2009), Los salarios del miedo. Mercado de trabajo y crecimiento económico en España durante el franquismo, Santiago de Compostela, Fundación 10 de Marzo.

Vilar Rodríguez, Margarita y Jerònia Pons Pons (2012), "The Introduction of Sickness Insurance in Spain in the First Decades of the Franco Dictatorship (1939-1962)", Social History of Medicine, 26 (2), pp. 267-287.

Zapata Ballón, Ernesto (1956), El seguro de enfermedad en América Latina, XII Asamblea General de la Asociación Internacional de la Seguridad Social. México, Secretaría General de la C.I.S.S.

Zschock, Dieter K. (1986), "Medical Care Under Social Insurance in Latin America", Latin American Research Review, 21 (1), pp. 99-122. 